\title{
Le mythe du bon sauvage Hottentot
}

\begin{abstract}
Two themes in the Voyage à l'intérieur de l'Afrique par le Cap de BonneEspérance by François le Vaillant. 1790, allracted our altention: firstly, the criticism of the colonial system, modelled on Denis Diderot's criticism, and secondly, the description of the Hottentot people according to the mythical vision of the tribes as suggested by Jean-Jacques Rousseau. A brief explanation of Diderot's anti-colonial ideas and of the myth of the 'noble Savage ' as suggested by Rousseau, will be followed by a critical analysis of the Voyage ... so as to prove that this work is in perfect agreement with the ideologies existing in France during the second half of the 18 th century and in line with the reformist notions of the thinkers who reconcile humanity and self-interest.
\end{abstract}

\section{Le Voyage à l'intérieur de l'Afrique par le Cap de Bonne Espérance de François le Vaillant}

En 1790 parut à Paris, le Voyage à l'intérieur de l'Afrique par le Cap de BonneEspérance, l'oeuvre présumée de François le Vaillant. Né en Guyanne hollandaise en 1753, d'un père alsacien exerçant la fonction de Consul de France en ce pays, François le Vaillant passa son enfance à Paramaribo. Sans doute estce là qu'il prit goût aux voyages et à l'onithologie. La première édition du récit de son voyage', fut accueillie avec enthousiasme par le public, bien qu'il fût rapidement prouvé que le texte avait été rédigé par Varon et Legrand d'Aussy

I Une deuxième édition revue et considérablement augmentéc par l'autcur, ornće de figurcs en taille douec, dont 8 n'avaient pas encore paru, fut éditée à Paris, l'an VI, e'cst à cette édition que nous nous référons dans le présent article.

Le Second voyage dans l'intérieur de l'Afrique dans les années 1789-85, paru chez Jansen à Paris, l'an III en 2 volumes in $4^{\circ}$ ou 3 vol. in $8^{\circ}$. Cette nouvelle édition ctait augmentée d'une carte d'Afrique et d'unc table des matières servant aux deux ouvrages de le Vaillant. 
d'aprés les notes personnelles de le Vaillant. ${ }^{2}$ En effet, on sait que ce demier, qui avait passé son enfance dans les forèts de la Guyane hollandaise et sa jeunesse en Afrique, était incapable d'écrire le français, mème s'il le parlait couramment. II est donc généralement admis qu'il eut recours à quelqu'un pour corriger ses épreuves et les enrichir de quelques réflexions politiques et philosophiques. Le texte que nous nous proposons d'analyser est donc un montage littéraire qui nous intéresse par les idéologies qu'il véhicule; en effet, Varon et Legrand d'Aussy, qui n'avaient jamais mis le pied en Afrique, éclairérent les observations du voyageur le Vaillant à la lumière des idéologies à la mode dans leur pays à la fin du $18 \mathrm{e}$ siècle. Nous désignerons par le nom de le Vaillant les scripteurs du Voyage ... .

Outre ses observations ornithologiques et scientifiques, le récit contient deux thèmes qui nous intéressent particulièrement: la critique du système colonial et une description mythique du peuple Hottentot.

\subsection{La critique du système colonial chez Denis Diderot.}

Notre analyse du Voyage ... démontrera, dans un premier temps, comment la critique du systéme colonial de le Vaillant est calquée sur celle de Denis Diderot, telle que nous la trouvons dans Le supplément au Voyage de Bougainville, paru en 1773, et dans l'édition de 1780 de 1 'Histoire des Deux Indes. ${ }^{3}$ Diderot s'inquiétait de la menace que la présence des Européens faisait peser sur le monde sauvage à la vie rude et fragile. II était convaincu, en effet, qu'il n'y avait de bonheur possible pour l'espèce humaine que dans un état social où "il n'y aurait ni roi, ni magistrat, ni prètre, ni tien, ni mien, ni propriété mobilière, ni propriété "foncière, ni vices, vertus" (Raynal, 1783, IX: 295). Les abus de la colonisation sont entraînés par la notion de la propriété. L'arrivée des Européens, qui viennent s'approprier la terre, apporte avec eux les germes de la destruction. Le supplément contient une violente attaque contre la barbarie des civilisés et condamne le systéme colonial; l'esclavage auquel sont réduits les Tahitiens et les Hottentots, dépossédés de leur terre, est contraire à la nature. Diderot n'affirma jamais cependant que l'état sauvage était préférable à l'état policé, mais ce qu'il souhaitait

2 Varon est signalé comme le rédactcur des voyages de lc Vaillant et Legrand d'Aussy y aurait mis la "dernic̀re main": ce fait est confırmé par Hocfer dans la Nouvelle Biographie générale, de même que par V.S. Forbes dans lioneer Travellers in South Africa. Balkema, Cape Town, Amsterdam, 1965 et par J. Mciring dans The Truth in Masquerade, the Adventures of François le Vaillant.

3 L'abbć Guillaume-Thomas Raynal publia en 1770 rHistoire philosophique et politique des érablissements de commerce des européens dans les dewx Indes, qui voulait ĉtre une dénonciation des crimes du fanatisme catholique dans les colonics. Avec la collaboration de Diderot et d'Holbach, il en donna une ćdition augmentéc en 1780 qui fut condamnéc par le Parlement de Paris et brûlćc 
c'était que l'équilibre entre les avantages et les maux propres à chacun des états ne soit pas rompu et que l'homme naturel ne soit pas enchaîné par l'homme moral. Diderot dépeignit surtout la tyrannie des mauvaises lois, le bonheur de l'homme soumis à la loi de la nature, les plaisirs de la volupté innocente.

\subsection{La vision mythique des peuples sauvages chez Jean-Jacques Rousseau}

Nous verrons, dans un deuxième temps, que la description des moeurs des Hottentots est imprégnée du "Discours sur les origines de l'inégalité" et de "l'Essai sur l'origine des langues" de Jean-Jacques Rousseau, ouvrages dans lesquels ce dernier propose une vision mythique des peuples sauvages ou des peuples préservés de toute altération, vivant dans leur institution primitive et dans la simplicité de leur génie originel, alors qu'ils jouissent encore de l'innocence, de la liberté, de l'égalité et de l'ignorance. Il est important de préciser que cette vision est de nature mythique puisqu'elle est dictée par la méthode conjecturale chère à Rousseau. En effet, ce dernier, contrairement à la plupart des écrivains de son siècle, ne recourt pas nécessairement aux ressources de la connaissance empirique et discursive et il refuse aussi la perspective historique qui, selon lui, ne montre qu'une vision dégradante de l'humanité à travers des témoignages incertains. A la logique et à l'empirisme, il préfère la connaissance intuitive, la méthode de réflexion hypothétique et la conjecture. Il se propose d'écrire l'histoire mythique de l'humanité telle que son imagination la lui suggère. il reconstruit ainsi les structures originelles du monde et de l'humanité, de l'enfance et du langage dans ses oeuvres dominées par la vision mythique de l'àge d'or. ${ }^{4}$ L'âge d'or, tel que Rousseau le décrit, se situe dans un temps mythique intermédiaire entre la nature et la société, et présente un stade d'équilibre et d'harmonie dans l'évolution de l'humanité. Les sauvages d'Amérique et des Caraibes sont, selon Rousseau, encore proches de cet état de bonheur parfait. ${ }^{5}$ Selon Le Vaillant, les Hottentots qui ont choisi de préserver leur patrimoine naturel après l'arrivée des colonisateurs, ont gardé les vertus de l'àge d'or.

4 L'âge d'or est célébré par les poctes grecs ct latins et imaginé par cux. II s'agit du temps des origines de l'humanité sous le régne de Cronos, chez les Grecs, et de Saturne, chez les Latins, où les hommes vivaient dans le contentement et la paix, en jouissant de la fécondité du sol ct de tous les biens dispensés par la nature. Leur bonheur était dû à l'ignorance de la guerre ct à l'absenee du droit individucl de propriété.

9 Rousscau a applique sa vision mythique aux peuples du temps de la Barbarie et de l'Antiquité et aussi aux Montagnons qu'il a pu observer au cours de l'hiver 1830-31, dans le pays de Ncuchàtel et dont il parle dans le livre IV des Confessions. II applique aussi cette vision aux Valaisans dans la lettre XXIII dc la Nouvelle Hélotse. 


\section{L'image des Hottentots en France au 18e siècle}

Le Vaillant ne fut pas le premier à décrire les moeurs des Hottentots. En effet, la plupart des voyageurs qui avaient fait escale au Cap de Bonne-Espérance avaient parlé des Hottentots et avaient, à l'unanimité, donné pendant deux siècles un portrait physique répugnant de ces demiers ${ }^{6}$ dont les particularités anatomiques, la saleté repoussante, les usages particuliers et le langage bizarre suscitaient un profond dégoût ${ }^{7}$. Ceci n'empécha pas les penseurs français de s'y intéresser. Voltaire, par exemple, leur reconnut quelques qualités pratiques en les plaçant aux côtés des Canadiens, parmi les peuples "qui ont l'art de fabriquer eux-mèmes tout ce dont ils ont besoin"(1963, 1:23); l'abbé Yvon, auteur de l'article "Athée" de l 'Encyclopédies, hésitait mème à voir des athées dans ces peuples qui n'ont "ni temple, ni idoles, ni cultes mais qui reconnaissent une équité et savent le droit des gens". Raynal vantait leur concorde inaltérable et leur bienveillance. Diderot sembla excédé par la sensiblerie tant soit peu méprisante de ses contemporains: "je sais que vous vous éloignerez avec dégoût d'un homme emmailloté, pour ainsi dire, dans des entrailles d'animaux" (Raynal, 1783, IV: 239).

II faut se rappeler que pendant longtemps I'Afrique noire, la Nigritie, fut très mal connue dans son ensemble et que les principales relations venaient soit d'agents de compagnie, soit de missionnaires qui donnaient la plupart du temps une image particuliérement péjorative de l'Africain. Nous pensons au Voyage aux îles $d^{\prime}$ Amérique du père Labat, supérieur de la mission des Antilles, qui évalue dans son oeuvre parue en 1730 les qualités et les défauts des Africains, sans avoir mis les pieds en Afrique, en pensant aux esclaves des îles. 11 les considére comme un peuple d'un naturel trés mauvais qui ne peut trouver son salut que dans l'esclavage qui en fait des hommes et des chrétiens. Nous trouvons le mème genre de propos

6 Nous pensons notamment aux ouvrages du Hollandais Peter Kolb: Description du Cap de Bonne-Espérance (1741), ct Les mémoires sur les Hottentots (1743).

"Tablicr des Hottentotes, ablation d'un testicule, entrailles d'animaux autour des jambes, odeur repoussante, gloussements: tels sont les traits sous lesquels on les retrouve partout" (Duchet, 1977: 35). On désignc par tablier hottentot un trait génćtique qui se rencontre chez les femmes hottentotes, la macronymphic ou élongation des petites lèvres. Ce trait suscita au $18 \mathrm{e}$ siècle un intěrêt particulier, comme toutcs les singularités sexuclles d'aillcurs.

$8 \quad$ L'Encyclopédie, publiće sous la direction de Diderot, vulgarisa la pensée philosophique du $18 \mathrm{e}$ siécle. Cet ouvrage, qui devait ĉtrc une traduction de la Cyclopoedia de l'anglais Chambers devint l'Encyclopédie ou Dicionnaire universel des Sciences, des Arts et des Métiers. De nombrcux hommes de sciences, mathémathiciens, techniciens et philosophes français collaborèrent à la rédaction des 17 volumes de textes et 11 volumes de planches. dont l'édition commencée en 1751 se termina en 1765, tout en menant un combat acharnć contre la censure. 
dans l'Histoire et Description de Saint Domingue du pére Charlevoix, parue en 1750, et dans L'Histoire des voyages de l'abbé Prévost', 1732-39. C'est le "nègre" des Antilles qui est décrit dans ces ouvrages; transplantés en Amérique comme esclaves, les habitants de la Nigritie cessent d'ètre des Africains.

Ce n'est qu'aux alentours de 1760 qu'un intérêt plus sérieux se manifesta en faveur de l'Afrique, lorsque la crise du systeme esclavagiste amena les physiocrates à préconiser que la culture du sucre soit établie en Afrique.10 Alors apparut l'image rousseauiste rassurante d'un inonde sauvage heureux, jouissant de l'état de pure nature, où les hommes ne sont pas dénaturés et où le mal n'est qu'ignorance. Le naturaliste français Adanson, un des premiers à proposer de faire de l'Afrique une colonie de peuplement, dresse un inventaire des ressources naturelles du Sénégal et son Histoire Naturelle du Sénégal (1757) présente, comme l'a écrit M. Duchet, une image plus riante du paysage africain et de ses habitants,

l'image la plus parfaite de la pure nature qui rappelle au naturaliste l'idée des premiers hommes et le monde à sa naissance, une vision idyllique des heureux africains qui les absout du péché de barbarie, et leur ouvre la terre promise de la civilisation (1977: 47).

Les Hollandais, fascinés par leur colonie du Cap de Bonne-Espérance, décidèrent d'explorer l'arrière-pays. Le gouverneur Tulbagh organisa une expédition en 1752, et elle fut suivie de celle de Hendrik Hop en 1761. Celui-ci qui gagna la rivière Orange et le territoire des Namaques et publia Une nouvelle description du Cap de Bonne Espérance en 1778. De 1752 à 1753, l'abbé de la Caille séjourna au Cap et son Journal historique du voyage fait au Cap de BonneEspérance, paru en 1763, contient de nombreuses observations sur les Hottentots. Bernardin de Saint Pierre dans son Voyage à l'ille de France, dénonça la fable du tablier des femmes hottentotes, ce que confirma le naturaliste suédois Sparrman dans son Voyage au Cap de Bonne-Espérance et autour du monde avec le Capitaine Cook, et principalement dans le pays des Hottentots et des Caffres,

II fut aussi l'autcur du célèbre roman. L'Histoire du chevalier des Grieux et de Manon Lescaut, (1732-39)

10. Les physiocrates forment une école d'ćconomistes en France, dont les travaux se développent entre 1756 et 1780. Leur chef, Ic médecin François Quesnay, expose sa doctrine dans L'Tincyclopédie. La conception fondamentale des physiocrates ćtait celle de l'ordre naturel: la socićté, comme le monde physique et animal, est régie par des lois irrévocables qui tiennent à l'essence humaine, tout en étant l'expression d'un ordre providenticl. L'agriculture représentait à lcurs veux la principalc ressource économique et ils contribuc̀rent done à l'engoucment pour l'agriculture qui se manifesta en France dans la seconde moitić du $18 \mathrm{c}$ siècle. 
paru en 1787."1 Les Voyages à l'intérieur de l'Afrique de le Vaillant sont toutefois les plus fournis en détails concernant les Hottentots. Nous y reviendrons bientôt.

Il semble certain qu'une exploration plus systématique de l'Afrique amena, chez les scientifiques, comme chez les philosophes, un intérêt nouveau en faveur de l'Africain et leur fit mème parfois prendre conscience des dangers de la colonisation. De nombreux textes dénoncèrent les méfaits du commerce et de l'esclavage. L'Histoire des Deux Indes de l'abbé Raynal, qui connut 20 éditions entre 1881 et 87 , annonçait un changement de goût. Cette histoire est vue par J.C.Bonnet comme une "épopée du commerce, qui dénonce l'inhumanité du commerçant et la tyrannie des grandes puissances", comme "une histoire des forfaits de la colonisation, qui en souligne aussi les effets civilisateurs" (1984: 307). C'est Diderot qui, par son importante collaboration, donna à cette oeuvre un ton que n'oublièrent ni les révolutionnaires de 1789, ni Toussaint Louverture en Haïti. Outre les thèmes anticolonialistes, Diderot appela les peuples à la révolte comme il l'avait déjà fait dans le Supplément au Voyage de Bougainville en 1773, où il accusait la Hollande d'avoir rompu, par son commerce corrupteur, l'équilibre d'un monde harmonieux:

Fuyez, malheureux Hottentots, fuyez! Enfoncez-vous dans vos forèts. Les bètes féroces sont moins redoutables que les monstres sous l'empire desquels vous allez tomber. Le tigre vous déchirera peut-être, mais il ne vous ôtera que la vie. L'autre vous ravira l'innocence et la liberté. Ou si vous vous en sentez le courage, prenez vos haches, tendez vos arcs, faites pleuvoir sur ces étrangers vos flèches empoisonnées. Puisse-t-il n'en rester aucun pour porter à leurs citoyens la nouvelle de leur désastre (Diderot, 1969-1973, XV: 432).

La violence des vocables utilisés dans cet appel à la révolte montrent la colère de Diderot qui était très choqué par les abus des colonisateurs, perpétrés au nom de la notion de propriété et de la morale. Il est important de souligner le fait que Diderot en appelle à la concience des opprimés, ce qui était exceptionnel au 18e siècle. Ce texte fait écho à d'autres textes qui dénoncèrent les méfaits du commerce et de l'esclavage: l'histoire du nègre de Surinam dans le Candide de

11 M. Duchet nous signale que Raynal copia la Caille et que Buffon prit ses informations concernant les Hottentots du journal du Vicomte de Querhoent, qui les avaient lui-même reçues de Lord Gordon qui avait accompagné Patterson à l'intérieur du pays; la relation du voyage de Gorgon parut en 1790. Diderot rencontra Gordon en Hollande et l'interrogea au sujet des Hottentots. II n'y eut aucune mise à jour des articles 'Afrique' et Hottentot dans L'Encyclopédie (Duchet, 1977 : op. cit.:55). 
Voltaire, ch. XIX, et dans le roman futuriste de Mercier, le "singulier monument" de L'an deux mille quatre cent quarante (1771).12

\subsection{La critique du système colonial dans Le Voyage ...}

Le récit de le Vaillant nous semble être une synthèse de ces différentes opinions courantes en France dans la seconde moitié du 18e siècle en faveur de l'homme noir et contre le systéme colonial tel qu'il existait. Comme nous l'avons déjà annoncé, deux thèmes majeurs dominent cette oeuvre: la critique virulente des civilisés, c'est-à-dire des colonisateurs, et, en antithèse, l'apologie du bon sauvage.

Commençons par analyser la critique des nations policées, éparpillée à travers les deux volumes du Voyage à l'intérieur de l'Afrique. C'est d'abord le système colonial lui-même qui est remis en question: l'organisation d'une colonie devrait être planifiée par une compagnie de commerce "aux grandes vues" et non confiée à "l'indolence d'un absurde colon, accoutumé à voir dans une légère difficulté des obstacles insurmontables" (I: 192). Une bonne exploitation des terres du Cap et de l'arrière pays, organisée par une compagnie, attirerait des colons "intelligents" et non des gens intéressés à "étouffer tout germe qui tendrait à diminuer leur profit". La mauvaise gestion de la colonie est due en grande partie à la responsabilité du gouvemeur apathique et indolent, ayant consenti à s'expatrier pour faire fortune, leurant son gouvernement "tout en faisant un étalage grossier de sa fortune"; ainsi, de gouverneur en gouverneur, "le citron est cent fois pressé" (I: 197).

Ensuite le Vaillant prend la défense de l'homme sauvage sur qui les nations policées feraient bien de prendre exemple. De nombreux passages expriment son admiration pour les Hottentots et, en mème temps, son désaccord avec les vues exprimées par d'autres:

un de ces humains dont les nations policées ne parlent qu'avec horreur ou mépris; que sans les connaître, elles regardent comme des êtres atroces, le rebut de la nature; en un mot un sauvage de I'Afrique, un Caffre, un

12 "Le singulier monument" est le mémorial des horreurs de vingt siécles où chaque nation demande pardon à l'humanité pour les crimes qu'elle a commis. A droite de ce monument se trouve sur un "magnifique piédestal un nègre, la tête nue, le bras tendu, l'oeil fier, l'attitude noble, imposante. A ses pieds on lisait ces mots: Au vengeur du nouveau monde" (Mercier, 1971: 201). Ce Spartacus noir, fait penser à Toussaint Louverture, chef des insurgés de Saint-Domingue de 1796 à 1802, qui tenta de devenir maître de lî̀le en chassant les espagnols. Fait prisonnier par le général Brunnet, il mourut en France, au Fort de Joux. Il s'adressait ainsi à Bonaparte: "le premier des noirs au premier des blancs". 
Hottentot.(..). On n'a jusqu'ici que des notions bizarres ou d'absurdes romans sur le pays singulier que je parcours (l: 212).

Un autre cliché de l'époque est défendu: si les Caffres, dépeints par "d'ignorants écrivains" comme "plus altérés de sang que d'autres sauvages", sont devenus tels, c'est à cause des colons qui, par leurs odieux traitements "les ont portés davantage à la guerre". La vengeance est de droit naturel et il est simple de prouver par les faits plutôt que par le raisonnement "lequel est le plus barbare d'un sauvage ou d'un blanc" (1: 243). Cette opinion rejoint les arguments des anticolonialistes et antiesclavagistes: la méchanceté des sauvages est due à une altération de leur naturel provoquée par les cruautés des Européens qui les ont corrompus. ${ }^{13}$ Le Vaillant, gràce à son comportement irréprochable, est adopté sans difficulté par les sauvages qui ne font plus rien sans le consulter.

Un autre reproche est adressé aux nations policées, celui d'avoir rompu l'équilibre en désunissant les Hottentots pour les précipiter dans l'esclavage et la décadence, dont le processus suit le schéma proposé par Jean-Jacques Rousseau. ${ }^{14}$ Celui-ci voyait trois étapes dans le devenir inéluctable de l'homme: l'état de pure nature, l'àge d'or et l'éloignement de la barbarie de l'àge d'or. La premiêre étape, l'état de pure nature est atemporel, antérieur à l'éclosion de l'histoire, à la constitution des sociétés au temps où l'homme, dispersé dans l'univers, ignorait les principes de l'association et de la communauté, vivant replié sur son être en vue de sa conservation.

La deuxième étape, l'âge d'or, est l'état intermédiaire entre la nature et la société, passage impossible à déterminer dans la durée historique, de l'innocence de l'état de nature à la fondation de l'Etat civil. C'est l'époque où les peuples renoncent à la dispersion spatiale pour se grouper en hordes. Dans ce temps de la vie pastorale, l'homme s'adonne à l'élevage qui lui procure subsistance, habitation et habillement; il vit en autarcie sans la propriété, donc dans la liberté, et dans l'indolence puisque la Nature met tout à sa disposition. Les besoins restreints ne

13 Les réflexions de Le Vaillant rappellent les adieux du vieillard dans le Supplément au Voyage de Bougainville, "Regarde ces hommes, vois comme ils sont droits, sains, robustes. Regarde ces femmes, vois comme elles sont droites, saines, fraîches et belles (...). A peine t'es-tu montré parmi eux qu'ils sont devenus voleurs. A peine es-tu descendu sur notre terre qu'elle a fumé de sang" Diderot, 1969-1973, X: 204).

14 Nous y avons fait allusion plus haut, Rousseau propose ces ćtapes dans Ic "Discours sur l'origine de l'inégalité parmi les hommes", 1753, pour la rédaction duqucl il cut recours aux relations des voyageurs qui avaient décrit la vie sauvage: l'Histoire des Antilles du père de Tertre, 1667, Les mémoires sur les Holtentots de Peter Kolbe, 1743, et IHistoire des voyages de l'abbé Prévost, qui paraissait depuis 1746. Certains points sont repris dans l'"Essai sur l'origine des langues". 
nécessitent pas l'organisation d'une communauté sociale soumise aux contraintes du travail et de la servitude. Rousseau associe ce temps mythique des peuples barbares à l'éclosion des désirs, à l'apparition du langage.

La troisième étape est celle où les peuples s'éloignent de la barbarie de l'âge d'or par la pratique de l'agriculture et de la métallurgie. La reconnaissance du droit de propriété entraîne l'inégalité sociale et la constitution de l'Etat civil. Cet éloignement, Rousseau le juge fatal parce qu'il entraîne la dégradation et la décadence de l'humanité corrompue par la pénétration des arts, des sciences, de la philosophie, par l'envahissement de la richesse et du luxe. A ce stade de l'humanité, Rousseau propose deux types de peuples: d'un côté, les peuples barbares et sauvages qui restent attachès à la liberté et à l'égalité, tout en respectant les traditions qui les maintiennent en contact avec le monde et la nature, et, d'un autre côté, les peuples savants et policés qui, aprés avoir adopté les progrès de la culture, tombent dans l'esclavage civil (1832, 1-2:11).

C'est l'arrivée des Hollandais qui, d'après Le Vaillant, a précipité les Hottentots de l'âge d'or dans l'escalavage civil. Riebeek, en politique adroit, employa toutes "les voies détournées" pour s'attirer la bienveillance des sauvages:

Il couvrit de miel les bords du vase empoisonné. Gagnés par de cruels appats, les maitres imprescriptibles de toute cette Afrique, les sauvages, ne virent point tout ce que cette profanation coupable leur enlevait de droits, d'autorité, de repos, de bonheur. Indolents par nature, vrais cosmopolites et nullement cultivateurs, pourquoi se seraient-ils inquiétés que des étrangers fussent venus s'emparer d'un petit coin de terre, inutile et souvent inhabité? (Vaillant, I: 255) (Nous soulignons).

Les ternes utilisés par le scripteur dénoncent, comme chez Diderot, le droit de propriété que s'arrogent les colonisateurs et qui contient en germe la destruction des terres et des hommes. Les étrangers offrent aux Hottentots "deux amorces séduisantes" (l'alcool et le tabac) dont les conséquences furent désastreuses: "plus de liberté, plus de fierté, plus de nature, plus de Hottentots, plus d'hommes" (I: 256). La colonie prospc̀re et rien n'échappe aux progrès de "l'ambitieuse cupidité":

On jugea, comme cela se pratique toujours, que la loi du plus fort était un titre suffisant pour s'étendre à volonté; cette logique rendit nuls ceux de la propriété, si sacrés et si respectables; on s'empara indistinctement, à plusieurs reprises, au-delà même des besoins, de toutes les terres (I: 257).

C'est alors que les Hottentots se divisent et qu'apparaissent deux types de peuples (selon le schéma de Rousseau). Ceux que l'élevage intéresse encore gagnent les montagnes du Nord et du Nord-Est, les autres, ruinés par le tabac et l'alcool restent, tout en renonçant à leurs moeurs ainsi qu'à leur "antique et douce 
origine". Ils vendent leurs services aux agriculteurs blancs qui se déchargent "des travaux pénibles sur ces infortunés Hottentots de plus en plus 'dégradés et abàtardis' (I: 258). Ils perdent à jamais ce que Rousseau appelle l'innocence et la quiétude, contraints d'adopter les progrès de la culture, ils se trouvent réduits à l'esclavage civil $(1832,1-2: 11)$. Au chef de leur choix succède un gouverneur, le parfait fonctionnaire de la tyrannie exercée par le gouvernement et les colons. Tout au long du Voyage, Le Vaillant raconte les atrocités commises par ces derniers, les commandos de représailles, tous les abus que permettent l'éloignement et l'isolement. Tel est le sort des Hottentots du Cap, à ne pas confondre avec les Hottentots (restés) sauvages, les jakals Hottentots. ${ }^{15}$

\subsection{La vision mythique du peuple hottentot}

Ce sont les moeurs des Hottentots restés sauvages en se réfugiant dans l'arrière pays que Le Vaillant décrit avec un enthousiasme quelque peu renforcé par l'attrait qu'il éprouve pour la belle hottentote Narina, une jeune fille de 16 ans dont il fait le portrait suivant:

je lui trouvai la figure charmante; elle avait les plus fraîches et les plus belles dents du monde; sa taille élégante et svelte, et les formes amoureuses de son corps, auraient servi le pinceau d'Albane. C'était la plus jeune des Grâces sous la figure d'une Hottentote (I: 366).

La description physique des Hottentots prend exactement le contre-pied de celles de Kolbe, Sparrman etc, qui insistaient sur la laideur et l'odeur repoussante des sauvages. Les femmes luisantes et fraîchement boughouées ${ }^{16}$, les jeunes gens, à la taille bien dessinée, à l'air et aux formes nobles, deviennent de beaux sauvages, comme les Tahitiens décrits par Diderot dans le Supplément au voyage de Bougainville (voir note 13).

Le peuple Hottentot est riche des vertus prédominantes de l'homme naturel telles que Rousseau les avait décrites: la liberté, l'égalité, l'innocence et l'ignorance. Dans l'âge pastoral le partage des terres n'existe pas et l'égalité des biens est générale, sans le droit à la propriété, il n'y a ni riche, ni pauvre:

Dans un pays oủ tous les individus sont égaux en naissant, pourvu qu'ils soient hommes, toutes les conditions nécessairement sont égales, ou plutôt il

15 Appelés ainsi parce qu'ils portaient le jakal (un morceau de peau d'animal), alors que le Hottentot de la côte s'habillait à l'européenne.

16 Frottées à la graisse et saupoudrées de poussière rouge (boughou) qui portc, d'après Le Vaillant, "une odeur assez agréable", alors que les autres voyageurs parlent de puanteur atroce. 
n'y a point de conditions; le luxe et la vanité, qui dévorent les fortunes et leur font éprouver tant de variations, sont nuls pour les sauvages; bornés à des besoins simples, les moyens par lesquels ils se les procurent n'étant pas exclusifs, peuvent être et sont effectivement employés par tous (Vaillant, II: 58).

La nation hottentote est libre et brave, fidèle à la simplicité primitive, à l'innocence originelle. Elle ne semble divisée par aucune exigence contradictoire. Enfin sa vertu majeure est l'ignorance dans un monde qui n'est corrompu ni par les inventions de la science, de la culture, des arts et des lettres, ni par la connaissance philosophique, mais qui a le sens des limites humaines:

Mortels heureux, (...)conservez longtemps cette précieuse ignorance; mais vivez ignorés! Pauvres sauvages, ne regrettez point d'être nés sous un ciel brûlant, sur un sol aride et désséché qui produit à peine des bruyêres et des ronces;(...) détruisez, effacez jusqu'aux moindres traces de cette poussière jaune qui se métallise dans vos ravines et dans vos roches; apprenez qu'elle est le fléau de la terre, la source de tous les crimes, et redoutez surtout l'approche d'un Amalgro, d'un Pizarre, d'un Fernand Cortès, et surtout l'étole ensanglantée ${ }^{17}$ des Vanverdes. Dans l'état de nature, l'homme est essentiellement bon; pourquoi le Hottentot serait-il une exception à la règle? (2: 122)

Dans ce texte, qui rappelle la folie destructrice de la conquête espagnole, il est fait allusion à une autre qualité attribuée par Rousseau aux peuples vivant dans la simplicité de leurs origines, l'ignorance:

Il y a une autre sorte dignorance raisonnable, qui consiste à borner sa curiosité à l'étendue des facultés qu'on a reçues;(...) une douce et précieuse ignorance, trésor d'une âme pure et contente de soi, qui met toute sa félicité à se replier sur elle-même, à se rendre témoignage de son ignorance (Rousseau, 1832, 3: 193).

De nombreux passages décrivent les traditions des Hottentots qui semblent immuables mais que le gouvernement colonial anéantira inéluctablement puisqu'un peuple ne peut garder son intégrité que dans la stabilité et la permanence, en restant fidèle à ses traditions et en respectant ses coutumes, ainsi que l'avait écrit Rousseau (1832:1-2:34):

Le moindre changement dans les coutumes, fût-il même avantageux à certains égards, tourne toujours au préjudice des moeurs. Car, les coutumes

17 Il cst intércssant de remarquer que le seriptcur n'cmploie pas le terme ćtendard ou drapcau mais cclui d'ćtole, qui désigne un instrument sacerdotal, faisant ainsi allusion aux missionnaircs qui coliaborcrent à toutes les occupations coloniales ct imposćrent bien souvent par la violence le christianisme aux colonisés. 
sont la morale du peuple; et dès qu'il cesse de les respecter, il n'a plus de règle que ses passions, ni de frein que les lois, qui peuvent quelquefois contenir les méchants, mais jamais les rendre bons.

Comme tous les peuples libres, les Hottentots ont le sens inné de la justice, pratiquent l'hospitalité et honorent la vertu. De nombreux anecdotes tendent à prouver leur supériorité morale sur les Européens (en ce qui concerne l'instinct maternel, la fidélité conjugale etc) (Vaillant, 2: 50 et suivantes), prenant encore une fois le contrepied des "balivernes" écrites par Kolb et qui furent reprises par "d'autres ignorants". "Je ne connais point de voyage sur l'Afrique, écrit le Vaillant, qui ne soit entaché des absurdes rêveries de Kolbe" (2: 55), dont les erreurs viennent de ce qu'il a cru les racontars des colons sans les vérifier. ${ }^{18}$

Enfin, comme tous les bons sauvages, les Hottentots ont le sens de la fête et de la musique:

Tout près de la nature, et sous sa garde immédiate, le sauvage n'a rien besoin de nos orchestres bruyants et bien harmonieux pour s'exciter, dans les fètes, aux vives démonstrations du plaisir et de la joie; la modulation bornée et monotone de sa musique lui suffit,et je crois même qu'il s'en passerait volontiers, et n'en sauterait pas moins bien (2: 107).

Tels sont les peuples ou tels lui ont-ils paru "dans toute l'innocence des moeurs et de la vie pastorale" offrant encore l'idée de l'espêce humaine dans son enfance (vol. 2: 120).

Le paysage africain devient l'image la plus parfaite de la pure nature, "riant séjour", "agréable solitude", "paysage charmant", la vision "champêtre", tels sont les vocables bucoliques utilisés par l'auteur.

\subsection{Les limites de l'anticolonialisme de François le Vaillant}

Loin d'être libéré de l'idée de la supériorité de l'homme blanc et de sa mission civilisatrice, le Vaillant explique aux voyageurs comment gagner la confiance des sauvages. Il ne faut surtout pas prendre modêle sur le capitaine Cook dont la mort est due à son tempérament altier et à l'indiscipline de son équipage qui

18 Dans son désir de présenter les Hottentots sous un jour plus humain, le Vaillant semble vouloir aussi detruire certaines légendes d'ordre sexuel par exemple, comme le tabier des Hottentotes, qui en faisaient des monstres. Kolbe, de nationalité hollandaise, avait passé longtemps pour un excellent auteur, sans doute sur la foi de Prévost ct de Rousscau qui le suivent aveuglément. La Caille et Buffon le démentiront (Duchet, 1977: 95) 
s'attira l'inimitié des insulaires (1: 126). ${ }^{19}$ II faut se montrer humain, bienfaisant, ne jamais abuser de la faiblesse des sauvages, ne leur inspirer aucune crainte, et surtout ne rien faire qui puisse faire perdre à leurs yeux "l'utile supériorité" qui les avaient éblouis, ne jamais utiliser la force mais au contraire les respecter, les aider, les protéger. Dans ce discours, qui en appelle au bon sens et à la conscience des colonisateurs, l'homme blanc reste celui qui est parfait et sa perfection lui accorde de civiliser autrui au nom de sa supériorité. Diderot avaient défendu exactement la même idée dans "Les principes que doivent suivre les Français dans l'Inde, s'ils parviennent à y établir leur considération et leur puissance":20 l'organisation des colonies doit se faire dans l'honnêteté et le respect de l'autre, le colonisateur doit être juste et humain, fidéle aux traités conclus:

Tant que vous séparerez le bien de la nation qui vous aura reçus, de votre propre utilité, vous serez oppresseurs; vous serez tyrans; et ce n'est que par le titre de bienfaiteur qu'on se fait aimer.(...) L'ouvrage de la sagesse n'est pas éternel: mais celui de la folie s'ébranle sans cesse, et ne tarde pas à crouler. La première grave ses caractères, ses caractères durables sur le rocher; la seconde trace les siens sur le sable (Diderot, 1969-1973, XV: 454).

Cependant le Vaillant ne pressent pas comme Diderot l'inévitable libération des peuples colonisés, il craint tout au plus qu'une révolte ne vienne des "basters blancs , qui inondent actuellement les colonies" et qui sont d'un naturel hardi et vindicatif parce que "le sang de l'Européen circule dans leurs veines" (2:140). Le Vaillant pensait qu'il aurait fallu favoriser le métissage des Hottentotes avec les noirs plutôt qu'avec les blancs, au nom de principes d'hérédité plus que douteux: l'enfant héritant souvent des défauts de son père, risque d'être vindicatif et intolérant si son géniteur est blanc; dans le cas contraire il sera doux, indolent, bon comme son père noir $(2: 141)$.

\section{Le réformisme des penseurs du 18e siècle}

Nous constatons, en conclusion, que l'idée de l'aliénation du sauvage par la colonisation domine le texte du Voyage ... en une vision conforme à l'idéal de la

19 Ce passage nous rappelle les adicux du vicillard tahiticn s'adressant à Bougainville: "Et chef des brigands qui t'obéissent, écarte promptement ton vaisseau de notre rive. Nous sommes innocents. nous sommes heureux, tu ne peux que nuire à notre bonheur. Nous suivons le pur instinct de la nature et tu as tenté d'effacer de nos âmes son caractère" (Diderot, Supplément, op. cit.).

20 Dans ec textc, qui offre un programme pour réformer et aménager le mode français de colonisation, Diderot prévoit aussi l'inćluetable liberation des peuples "parce que la folic coloniale ne construit que sur du sable". 
Révolution. Nous y trouvons également la dénonciation de l'esclavage et de la violence coloniale. Mais ces réflexions expriment aussi l'idée d'une disparition du monde sauvage à cause de ses propres faiblesses. II ne s'agit pas d'un véritable anticolonialisme qui revendiquerait la destruction du systéme colonial. Comme celui des penseurs du $18 \mathrm{e}$ siècle, le réformisme de le Vaillant n'est qu'humanitaire: il faut affranchir les esclaves, civiliser les peuples sauvages, concilier humanité et intérêt, c'est-à-dire composer avec le système dont la destruction supposerait un ordre économique différent, un nouvel équilibre commercial et une révolution politique. Au malheur des sauvages et aux injustices perpétrées contre eux, on ne propose qu'un remède: les civiliser. C'est ce que $M$. Duchet appelle le fondement moral d'un humanisme de la conquête, la preuve de ce que l'anticolonianisme des philosophes est une illusion (Duchet, 1977:21); remédier aux abus signifie contribuer au maintien de l'ordre établi. Les réformes proposées visent à assurer l'équilibre du systéme dans le cadre d'une économie marchande de production et de libre circulation des biens. Le principe des bons maîtres et des bons colons, qui doivent respecter les bons sauvages, érige le paternalisme en principe politique. Dans l'anthropologie du $18 \mathrm{e}$ siècle, l'homme civilisé reste le plus intéressant et civiliser le sauvage est l'élément le plus important de l'idéologie coloniale qui connaîtra le développement que l'on sait au 19e siècle

II était courant au $18 \mathrm{e}$ siècle que les récits des voyageurs fussent influencés par les écrits des savants, comme l'Histoire naturelle de Buffon, par exemple, ou par ceux des philosophes. II nous semble évident que les scripteurs du Voyage dans l'intérieur de l'Afrique, sans aucun doute Varon et Legrand d'Aussy, qui ont rédigé le texte à partir des observations de Le Vaillant, avaient lu Buffon, l'Encyclopédie, Rousseau et Diderot, les philosophes qui eux-mêmes avaient lu les ouvrages des savants qui n'avaient jamais mis le pied en Afrique et les récits des voyageurs qui ne vérifiaient pas toujours les dires des colons. C'est ainsi, sans doute, que s'inventèrent des stéréotypes comme le Persan railleur, le sage Egyptien et bien sûr le bon Sauvage, c'est-à-dire l'utopie du peuple proche de la rectitude des lois naturelles. Le mérite de le Vaillant cependant est d'avoir observé avec humanité et bienveillance les Hottentots, auprès desquels il passa plusieurs mois, et d'avoir transformé en un peuple mythique ces hommes méprisés par la plupart des voyageurs européens.

\section{References}

Adanson, M. 1757. Histoire Naturelle du Sénégal. Paris : Bauche. Bonnet, Jean-Claude. 1984. Diderot, textes et déhats. Paris: Poche.

Charlevois, M. 1750. Histoire et Description de Saim Domingue. Paris. 3 Vol.

Diderot, Denis. 1969-1973. Oewvres Complètes. Paris: Club français du livre, 15 vol.

Duchet, Michèle. 1977. Anthropologie et Histoire ant siècle des /.umières. Paris: Flammarion.

Forbes, V.S. 1965. Pioneer Travellers in South Africa. Cape Town : Balkema

Hoefer, J. 1860. La nowvelle biographie générale. Paris : Didot. 
Kolb, P. 1741. Description du Cap de Bome-Espérance. Amsterdam : L. Catuffe. 3 Vol.

Kolb, P. 1743. Les mémoires sur les Hottentots. Amsterdam : L. Catuffe.

Lacaille, N.L. 1763. Journal Historique du voyage fait Cap de Bonne-Espérance. Paris Guyllin.

Meiring, J. 1973. The Truth in Masquerade. The Adventures of Francois le Vaillant. Cape Town : Juta.

Mercier, Louis Sébastien. 1971. I'an 2440. Réédition préfacée et annotée par R. Trousson, Bordeaux: Ducros.

Raynal, Guillaume-Thomas, abbé. 1783. L'Histoire philosophique et politique des établissements et du commerce des Européens dans les Deux Indes. Neuchâtel-Genève, 10 vol.

Rousseau, Jean-Jacques. 1832. Oeuvres Complètes. Paris: Armand-Aubrée.

Sparman, A. 1787. Voyage au Cap de Bonne-Espérance et autour du monde avec le Capitaine Cook, et principalement dans les pays des Hottentots et des Caffres. Paris : Buisson

Vaillant le, François. An VI (1790). Voyage à l'imtérieur de l'Afrique par le Cap de Bomme Espérance. Paris : Leroy; $2^{\mathrm{e}}$ ed. 1798, Paris : Deray.

Voltaire, François-Marie. 1963. Essai sur les moeurs. Paris: Garnier, 2 vol. 
